\title{
Fits, Tantrums, and Rages in TS and Related Disorders
}

\author{
Cathy L. Budman ${ }^{1,3} \cdot$ Miri Rosen $^{2} \cdot$ Sana Shad $^{3}$
}

Published online: 8 August 2015

(C) Springer International Publishing Switzerland 2015

\begin{abstract}
Tourette syndrome (TS) is a neuropsychiatric disorder of childhood onset characterized by motor and phonic tics. In the majority of cases, psychiatric comorbidity complicates TS, manifesting with a wide range of affective and behavioral disturbances. Aggressive symptoms are relatively common in TS and have adverse impacts on family functioning and quality of life. The etiology of such symptoms may reflect a complex interplay among neurobiological, genetic, and environment factors. This paper provides an update on aggressive symptoms in TS with focus on explosive outbursts or "rages" and self-injurious behaviors. Recent studies highlight the association between aggression and common psychiatric comorbidities as well as the role of psychosocial influences. Aggressive symptoms in TS appear associated with tic severity and certain psychiatric comorbid disorders including attention deficit hyperactivity disorder (ADHD), obsessivecompulsive disorder (OCD), and mood disorders. Although still limited, pharmacological and non-pharmacological evidence-based treatments are emerging.
\end{abstract}

This article is part of the Topical Collection on Tourette's Syndrome

Cathy L. Budman

Cbudman@nshs.edu

Miri Rosen

MRosen13@nshs.edu

Sana Shad

sshad@nshs.edu

1 Hofstra North Shore-LIJ School of Medicine, North Shore-LIJ Health System, 400 Community Drive, Manhasset, NY 11030, USA

2 Division of Child and Adolescent Outpatient Psychiatry, Zucker Hillside Hospital, 75-59 263rd St, Glen Oaks, NY 11004, USA

3 Division of Outpatient Psychiatry, North Shore University Hospital, 400 Community Drive, Manhasset, NY 11030, USA
Keywords Tourette syndrome $\cdot$ Explosive outbursts $\cdot$ Rages · Aggressive symptoms $\cdot$ Disruptive behaviors

\section{Introduction}

Tourette syndrome (TS) is a neurodevelopmental disorder of childhood onset characterized by waxing and waning of motor and phonic tics present for over 1 year [1]. Meta-analyses of population-based prevalence studies estimate TS in 0.52 $0.77 \%$ of youth [2-4]. Tic severity appears to peak between ages $10-12$ years and often declines significantly by late adolescence, although a minority may experience persistent and disruptive tics into adulthood [5, 6, 7•]. Co-occurring psychiatric conditions are extremely common and associated with greater overall morbidity in TS [8-10]. Based on the largest comprehensive study of psychiatric comorbidity in TS to date, the estimated lifetime prevalence of any psychiatric comorbidity in TS is $85.7 \%$ with over half suffering from two or more psychiatric disorders [11・•]. Most individuals with TS develop psychiatric symptoms during childhood and meet diagnostic criteria for obsessive-compulsive disorder (OCD) or attention deficit hyperactivity disorder (ADHD), mood disorders, nonOCD anxiety disorders, and disruptive behaviors also occur with increased frequency $[11 \bullet \bullet]$.

Aggressive symptoms and problems with anger control appear common in TS and cause considerable morbidity $[12-15,16 \bullet \cdot$. Results from a large international survey of TS specialists indicated that $37 \%$ of all TS patients reported anger control problems at some point in their life and $25 \%$ reported currently experiencing anger control problems [17]. In a study of 58 Swedish schoolchildren with TS, teachers reported $35 \%$ of the children had major problems with aggression [14]. Parents and teachers reported significantly higher ratings of anger and externalizing problems in a clinical 
study of 25 children with TS when compared with that in healthy controls $[18 \bullet]$. Early literature on aggressive symptoms in TS was limited primarily to case series, retrospective studies, and relatively small clinical trials; limitations of recent large epidemiological studies include varying methodologies for assessing and characterizing aggressive symptoms and their severity [19]. Meanwhile, clinicians face the need to better recognize, evaluate, and appropriately treat these common and highly disruptive symptoms in their TS patients. This paper will briefly discuss predatory/non-reactive and nonpredatory/impulsive aggression, describe the phenomenology of aggressive symptoms in TS and associated psychiatric disorders, consider known and potential etiologies of such symptoms, and highlight the emerging evidence-base for pharmacological and non-pharmacological treatments of affected individuals.

\section{Pathological Aggression}

Some aggressive behaviors occur during normal development in early childhood. "Temper tantrums" are episodes of severe frustration and anger that may involve screaming, falling to the floor, biting, or head banging. These behaviors are most commonly observed in children ages 3-5 years; up to $83.7 \%$ of preschoolers were found to have temper tantrums [20]. Temper tantrums become much less frequent as children mature and develop the ability to inhibit unacceptable impulses and actions [20,21]. More extreme aggression towards people or animals and severe destruction of property are associated with later development of conduct disorder [22]. Pathologic aggression describes aggressive behaviors that are age inappropriate, inapt for social context, or excessive in intensity, duration, and frequency [23]. Up to $60 \%$ of referrals to outpatient child psychiatric clinics are explicitly for evaluation and treatment of aggression [22, 23].

Aggressive behaviors are classified as proactive/"non-impulsive" or reactive/"impulsive" depending upon key features. Proactive or "non-impulsive" aggression is characterized by aggressive behaviors that are premeditated and performed to attain a specific goal, typically accompanied by low levels of physiological arousal and limited guilt or regret [24, 25]. Reactive aggression or "impulsive aggression" is spontaneous, without obvious intent or organization, usually triggered by acute frustration, and is accompanied by high levels of physiological and emotional arousal [24, 25]. Causes of aggressive symptoms are diverse and may include environmental factors such as prenatal exposure to alcohol, drugs of abuse, and lead poisoning as well as psychosocial factors including poverty, malnutrition, and chaotic home environments [26]. Victims of physical, sexual, or emotional abuse are all at higher risk for symptoms of pathologic aggression [23, 26, 27].
Aggressive symptoms are frequent in other psychiatric conditions including autism spectrum disorder (ASD), ADHD, oppositional defiant disorder (ODD), conduct disorder (CD), alcohol and/or substance abuse disorders, bipolar disorder (BD), intermittent explosive disorder (IED), and the relatively newer DSM-V diagnostic entity "disruptive mood dysregulation disorder" (DMDD) [1]. Impulsive, unpredictable aggressive outbursts are cardinal symptoms of IED and may include verbal tirades or physically aggressive displays that are grossly out of proportion to the preceding precipitant or stressor causing distress, financial or legal consequences, and/or impairment of function. Such outbursts must occur twice a week for at least 3 months [1]. DMDD is also characterized by the onset of severe and recurrent developmentally inappropriate temper outbursts that occur three or more times each week for at least 1 year or more with onset in childhood; these outbursts are also grossly out of proportion in intensity or duration to the situation $[1,28]$. However, in contrast to IED, between outbursts children with DMDD experience a persistently irritable or angry mood, most of the day and nearly every day, present in at least two settings (e.g., at home, at school, or with peers), severe in at least one setting, and present for at least one or more years [1]. Increased irritability, disinhibited anger, and aggression can also occur in a number of other movement disorders such as Huntington's disease or Wilson's disease and from traumatic brain injury, dementias, or encephalitis [29,30]. Pediatric acute onset neuropsychiatric syndrome (PANS) characterized by both early onset OCD and tics may manifest with severe anxiety, emotional lability, irritability, highly oppositional behaviors, and aggression ([31]. PANS should be considered in any child with new, abrupt onset of OCD with or without tics symptoms showing aggression, particularly before making a diagnosis of IED or DMDD.

\section{Neurobiology of Aggression}

Pathological aggression can result from abnormal development or damage to areas of the brain involved in executive function and affective regulation [32]. The amygdala regulates aggressive behaviors by receiving sensory input from the thalamus and cortex; if an aggressive stimulus is present, the amygdala signals a response through the hypothalamus to the periaqueductal gray [33]. Reduced amygdala volumes, heightened reactivity to socially threatening stimuli, and dysfunction of the limbic prefrontal regions (i.e., orbitofrontal cortex) and of the ventral striatum have all been associated with aggression [25]. The major transmitters involved in the neuroanatomical loops that enable threat assessment and promote appropriate action (i.e., "fight" or "flight") include dopamine, serotonin, and norepinephrine [23, 25]. Imbalances of these neurotransmitters in the prefrontal cortex, or of 
excitatory glutamate and inhibitory gamma-aminobutyric acid in the subcortex, can lead to increased aggression [25, 27]. Increased testosterone levels and low cortisol levels are also associated with aggressive behaviors [23, 33].

\section{Aggressive Symptoms in TS}

Aggressive symptoms in TS encompass both non-impulsive and impulsive aggressive behaviors. Both clinically referred TS studies and investigations using community samples report similar highly disruptive aggressive symptoms that are primarily although not exclusively impulsive in nature [5, $16 \bullet \bullet, 34,35 \bullet$. This paper will highlight the two most commonly clinically encountered aggressive symptoms in TS: explosive outbursts and self-injurious behaviors (SIB). Some individuals with TS also describe urges to make insulting remarks or perform other socially disruptive, inappropriate behaviors [36, 37•]. While the relationship of such behaviors with explosive outbursts remains unclear, these non-obscene socially inappropriate symptoms (referred to by some investigators as "NOSIS") appear aggressive and may result in legal problems [37•].

\section{Explosive Outbursts/“Rages"}

Explosive outbursts (also referred to as "Rage Attacks") are severe and recurrent impulsive/reactive episodes of anger. These symptoms are a leading cause of morbidity in TS and have been reported worldwide [12, 17, 38]. Studies using clinically referred samples suggest that approximately 25$70 \%$ of individuals with TS also report problems with uncontrollable anger and, when present, cause significant disruption $[16 \bullet \bullet, 19,38-44,45 \bullet \bullet$. The onset of explosive outbursts in TS typically occurs during childhood and may herald a particularly agonizing period for family and peer relationships, often derailing normal psychosocial development and academic progress. The clinical phenomenology of explosive outbursts in TS has been explored using modified DSM-IV diagnostic criteria for IED $[13,35 \bullet, 38,40]$. Approximately $23-40 \%$ of clinically referred TS subjects report distressing behavioral symptoms of this type: sudden unpredictable anger, irritability, temper outbursts, and aggression but due to lack of systematic evaluation, their prevalence and etiology remain unknown [19].

Like IED, explosive outbursts in TS occur with little to no apparent provocation or are grossly out of proportion to the stressor [39]. Common precipitants include being reprimanded or corrected, feeling frustrated by failure to get one's way, or by confrontation with a change in routine or schedule [13, 38]. Also, like IED, explosive outbursts in TS may result in the physical mutilation and destruction of property or involve devastating verbal assaults on loved ones [39].
Explosive outbursts are sometimes preceded by a mental or sensory urge, almost always accompanied by heightened physiological arousal, typically experienced as uncontrollable and distressing, and frequently accompanied by subsequent shame with remorse [13]. This latter prosocial trait is in contrast to the limited empathy, more pervasive and enduring antisocial activities such as stealing, lying, and serious violations of rules that characterize those with Conduct disorder (CD) [1]. In addition, individuals with explosive outbursts and TS do not usually have a family history of aggressive, violent behaviors, arrests, or legal encounters as is typical with CD. Unlike DMDD, once the explosive episode concludes, there may be a sensation of increased "calm" or even fatigue, although a subset of children with TS and explosive outbursts appear to remain highly irritable [13]. Surveys of explosive outbursts completed by parents of children with TS report that these episodes occur most frequently at home and are most commonly directed towards a parent, usually the child's mother although less frequently towards siblings, pets, and property $[13,40]$. It appears that these episodes are much less likely to occur when the child with TS is at school or in other settings $[13,38]$.

Current evidence suggests that the etiologies underlying explosive outbursts in TS may be both phenotypically and genotypically diverse, resulting from a variety of psychological, biological, and environmental conditions [11••, 13, 19, $35 \bullet, 46]$.

\section{Self-Injurious Behaviors}

Self-injurious behaviors (SIB) comprise symptoms that are deliberate, repetitive infliction of self-harm without suicidal intent. SIB occur in 14-60\% of all patients with TS and have been reported to be correlated with tic severity, high levels of obsessiveness and hostility, and with increasing number of comorbid psychiatric disorders [16••, 17, 47-49]. These selfdirected aggressive symptoms include moderate self-injury by repetitive poking, pinching, slapping, biting, or more severe and complex aggressive behaviors directed towards self such as violent head banging, breaking fingers, extracting teeth, or causing facial fractures and may occur in even mild cases of TS [50]. A study examined nearly 300 subjects with TS revealed that mild/moderate SIB correlated with the presence of obsessive-compulsive symptoms (including aggressive obsessions or violent compulsions), with the presence of obsessive-compulsive disorder (OCD), and with the overall number of obsessions. Severe SIB correlated with variables that related to both affect and impulse dysregulation including episodic rages/explosive outbursts and increased risk-taking behaviors [48]. A large survey of clinicians worldwide who treat TS reported rates of SIB as higher in those TS individuals with comorbid ADHD; the age at onset of SIB was found to be 7.4 years in the TS+ADHD group, as compared with 10 years 
in the TS-ADHD group [51]. A clinical investigation of rage attacks in Japanese youth with TS found that subjects with aggression were also more likely to engage in self-injurious tics [38].

\section{Aggressive Symptoms in TS and Tic Severity}

Recent evidence points to a relationship between aggressive symptoms and tic severity. Phenotype analyses from participants in a large genetic study by Mathews et al. show that mild/moderate and severe SIB correlated with tic severity [48]. A subsequent analysis of 218 TS from this same international cohort demonstrated an association between worsening tic severity, lower age of onset of tic disorder, and increased aggressive behaviors [35॰]. Robertson et al. also reported a significant association between tic severity and the presence of aggressive behaviors in 578 clinically referred cases of TS [16••]. A recent clinical study of 101 clinical patients with TS showed a significant association between tic severity and higher levels of irritability [52••]. However, others have failed to find a direct relationship between tic type or severity and aggressive symptoms $[38,40]$.

\section{Aggressive Symptoms in TS and Co-occurring Psychiatric Disorders}

Increased risk for explosive outbursts and other aggressive behaviors in TS appear highly associated with co-occurring psychiatric disorders. Several studies have shown that aggressive behaviors in clinically referred cases of TS closely relate to comorbid OCD, ADHD, and ODD [13, 17, 19, 34, 35•, 39, $40,44,53 \cdot \bullet]$. A recent retrospective clinical study of 92 youth with TS showed elevated behavioral problems including aggression and emotional lability on Child Behavior Checklist (CBCL) when compared with healthy controls, and those with comorbid OCD, ADHD, or combined ADHD+OCD had significantly higher levels of disruptive behaviors than those with TS only [53・•]. There were no differences in behaviors between subgroups of TS youth with comorbid conditions [53・0]. Improved recognition and effective treatment of cooccurring psychiatric conditions in TS may reduce overall psychosocial stress, which in turn may reduce tic severity [54]. The net effect may result in improved quality of life and functioning with diminished frequency and intensity of explosive outbursts $[35 \bullet, 41,55]$.

\section{Aggression in TS and ADHD}

ADHD is the most common co-occurring psychiatric morbidity in TS, typically presenting prior to onset of tics and associated with considerable adverse effects on academic performance, peer relationships, and overall functioning in TS $[53 \bullet \bullet, 56 \bullet$. Among clinical populations, $60-80 \%$ of TS patients have comorbid ADHD [17, 43, 57], although frequencies are lower (i.e., 17-18\%) in population-based studies [58]. Hischritt et al.'s recent cross-sectional study of prevalence rates in 1374 individuals with TS reported $54.3 \%$ had cooccurring ADHD and that increased rates of comorbid TS and ADHD suggest a shared neurobiological and genetic basis $[11 \bullet \cdot]$. Some investigators suggest that ADHD symptoms emerging after tic onset may be part of the underlying tic syndrome [59]. Supporting this hypothesis, a factor analysis of 639 patients with TS identified ADHD and impulsivity, coupled with aggressive behaviors, as one of three significant factors that account for $48.5 \%$ of symptomatic variance in TS [44]. It has also been postulated that many of the disinhibited behaviors (including aggression) seen in TS are due to ADHD comorbidity and unrelated to TS per se [16*0]. Others suggest that until the mechanisms that underlie both ADHD and TS are better clarified, it may be preferable to consider ADHD and TS as "co-occurring" or "co-existing" conditions [60•].

Combined ADHD and TS commonly co-occurs with other comorbid disorders including OCD, mood disorders, ODD, and conduct disorders $[16 \bullet \bullet, 51,57]$. ADHD is also associated with sleep difficulties, a combination that may further increase risk for aggressive behaviors in TS. A clinical study of children with TS and/or ADHD showed that subjects who had more arousals from sleep were more likely to display conduct disorder, hyperactivity/immaturity, and restless/disorganized behaviors [61].

Disentangling overlapping symptoms from co-occurring multiple categorical psychiatric diagnoses has further complicated our understanding of aggressive symptoms in combined TS and ADHD. Furthermore, clinical phenotypes evolve during development as emphasized by findings from a retrospective study of the long-term clinical course of TS in 100 clinically referred patients who received semi-structured assessments and rating scales of tics, OCD, anxiety, and ADHD were re-assessed after 10 years [6]. This study demonstrated that all patients who presented with TS+ADHD at the onset of treatment showed a different clinical phenotype at 10-year follow-up: $62 \%$ were subsequently classified as TS-only, $35 \%$ as TS+OCD, and $2 \%$ were classified as TS+ADHD+ OCD. In the subjects who initially presented with TS+ ADHD+OCD, only $14 \%$ showed the same clinical phenotype at 10-year follow-up while the majority no longer displayed ADHD symptoms [6].

Much evidence shows that rates of aggressive behaviors do not differ between subjects with TS and healthy controls, whereas those with comorbid TS+ADHD demonstrate similar rates of aggression as subjects with ADHD alone [56••, 62-66]. Children with TS and ADHD show subtle deficits in emotional-processing tasks, particularly of anger, relative to healthy controls and TS-affected children without ADHD 
[67]. A clinical study that compared problems with executive functioning in children with ADHD, ASD, and TS relative to healthy controls found increased problems with regulating emotions in children with TS [68•]. A study that compared 80 adults with TS-only to 64 adults with TS+ADHD using clinical interview with standardized ratings revealed more depression, anxiety, and "maladaptive behaviors" including aggression in the TS+ADHD group [69]. Comorbid ADHD has also been associated with increased irritability in adults with TS $[52 \bullet \bullet]$.

\section{Aggression in TS and Disruptive Behavior Disorders}

ODD commonly co-occurs with TS and chronic tic disorders, presumably through its association with ADHD, and may precede development of CD [1,51]. A worldwide survey of 6805 cases submitted by TS specialists estimated $12.3 \%$ had comorbid ODD or CD [51]. In their large cross-sectional prevalence study, Hisrschtritt et al. estimated $29.3 \%$ prevalence of ODD and $3.2 \%$ prevalence of CD in individuals with TS [11••]. A study of 48 clinically referred children with TS and rage attacks that used structured interviews and rating scales revealed $42 \%$ met criteria for ODD [13]. However, it may be important to identify particular dimensions of ODD when examining its association with rage symptoms. In a clinical study of 135 children with TS, ODD was present in $54 \%$; irritability symptoms of ODD such as temper outbursts were associated with obsessive-compulsive behaviors but not ADHD symptoms [70]. In a study of 578 clinically referred TS cases including both youth and adults, CD was present in $14.5 \%$ and was significantly associated with comorbid ADHD and family history of aggressive behaviors [16••].

\section{Aggression in TS and OCD}

Depending upon methodology, estimated rates of obsessive thoughts and compulsive behaviors that reach threshold for clinical diagnosis of OCD range from 20-50\% [11••, 58, $71,72]$. Rates of OCD were $20-22 \%$ in the Avon longitudinal population-based study [58], whereas Hirschtritt et al. reported prevalence rates of $50 \%$ for OCD in TS using crosssectional structured diagnostic interviews $[11 \bullet \bullet]$.

In a Japanese study of 53 clinically referred TS patients, severity of obsessive-compulsive symptoms, not impulsivity, was associated with impairments in global functioning and the presence of obsessions involving aggression correlated with worsened global functioning [73•]. Youth with OCD without tics also experience explosive outbursts. Aggressive symptoms in OCD appear context specific: to prevent exposure to a distressing trigger, from exposure to an anxiety-provoking trigger, or as a result of disrupting ritual completion [74••]. These rage attacks, similar to those described in TS, appear to occur unprovoked or "out of the blue," although the actual trigger is frequently an unrecognized internal experience/ obsession $[74 \bullet \cdot]$. Storch et al. found that more than half of the 86 youth with OCD reported experiencing rage attacks [73•]. In this study, youth with OCD and rage attacks displayed elevated OCD symptom severity and greater functional impairment compared to controls. There was a higher incidence of disruptive behavior disorders in those with rage and $\mathrm{OCD}$, but no difference in rates of chronic tic disorders and ADHD [74••].

While most investigators believe explosive outbursts in TS are primarily related to ADHD symptoms, there is also evidence implicating an association with OCD in TS $[35 \cdot, 40]$. It is possible that such discrepancies reflect shared and synergistic processes underlying both OCD and ADHD [75].

\section{Aggression in Mood Disorders and TS}

Mood disorders, including major depression and bipolar disorder may be associated with aggressive behavior. Irritability is a common symptom of both, and usually precedes reactive aggression. Rage attacks may signal atypical presentations of a depressive disorder in children or adults [27]. Various depressive symptoms occur in $13-76 \%$ of TS patients attending specialist clinics $[57,71,76]$. Results from Hirschtritt et al. report that $26.1 \%$ of cases met diagnostic criteria for major depressive disorder $[11 \bullet \bullet]$.

These relatively high rates of depression in TS may reflect multiple factors, including the considerable burden of living with a chronic and socially stigmatizing disorder. Additionally, major depression in TS appears highly associated with OCD and ADHD [57]. Depression in TS has been associated with increased aggression; a study of 91 adults with TS treated at a specialty clinic, $34.1 \%$ reported aggression towards others and $24.4 \%$ described aggressive behaviors towards property [77]. In this study, aggression correlated with depression scores on the Beck Depression Inventory (BDI) and on the Mood Adjective Checklist (MACL) [77]. Aggressive symptoms may also be a risk factor for suicide. In a recent clinical study of 196 youth with chronic motor tic disorder (CTD), aggressive behaviors were associated with suicidal thoughts or preparatory actions [78].

\section{Medication Treatment of Aggression and TS}

The treatment strategy for pharmacological management of aggressive symptoms involves first identifying, prioritizing, and then targeting the primary underlying psychiatric condition(s) associated with aggression. The evidence base for treating aggressive symptoms in TS is generally weak, based primarily on small open-label studies or trials, using varying methodologies, and often combining pediatric and adults study subjects. Haloperidol, pimozide, and now aripiprazole 
are the only psychotropic medications with FDA approval for the treatment of tics in TS and have anti-aggression properties [79]. In addition, a variety of other agents including short and long-acting alpha agonists, mixed norepinephrine-serotonin reuptake inhibitors, atypical and typical antipsychotics, and anticonvulsants are used off label to target tics and associated comorbid psychiatric symptoms including aggression [80॰].

\section{Selective Serotonin Reuptake Inhibitors}

Selective serotonin reuptake inhibitors (SSRIs) are prescribed for treatment of depression, anxiety, or OCD in TS, although their efficacy for targeting aggression in children and adolescents remains unproven [27]. An 8-week open-label study of paroxetine in a clinically heterogeneous cohort of 45 children and adults with TS and explosive outbursts found $76 \%$ reported that rages significantly diminished or were absent on a mean dose of $33 \mathrm{mg} /$ day. Four patients discontinued paroxetine due to exacerbations of rage attacks and one patient experienced a hypomanic episode. No patient in this study met diagnostic criteria for major depression, although the majority of subjects had OCD, ADHD, or both [39]. Larger, randomized placebo-controlled trials are needed to evaluate these preliminary results. Activation, hypomania, and aggression are adverse effects associated with SSRIs that require differentiation from primary aggressive symptoms [81].

\section{Psychostimulants}

There is reasonable evidence that stimulant treatment reduces aggression in children with combined TS and ADHD and in those with comorbid ODD [82, 83]. In a double-blind placebo-controlled trial of immediate-release methylphenidate for the treatment of ADHD in 71 children age 6-12 years with TS or CTD), parent and teacher measures of oppositional defiant behavior and interpersonal peer aggression showed marked improvement with methylphenidate immediate release treatment [84]. Further analysis of this study population comparing 31 children + ODD + TS to 26 children with TS without ODD demonstrated that children with ADHD+ODD also showed comparable and significant decreases in teacher- and parentrated measures of peer aggression when treated with methylphenidate, and teacher-rated aggression decreased at lower dosages than in non-ODD children [85].

\section{Antipsychotics}

There is increasing evidence that some atypical antipsychotics may be useful in the treatment of TS-associated rage [86•*]. In a retrospective review of risperidone treatment of 49 patients with TS, 28 subjects age 5 to 18 years presented with aggressive behavior at baseline and $22(78.5 \%)$ showed decreased aggression scores at the end of the treatment period on a mean dose of $2 \mathrm{mg} /$ day. The most common side effects were weight gain and increased emotional lability [87]. In an 8-week treatment phase trial of olanzapine, 10 children age $7-13$ years with TS and a history of aggressive behavior showed significant decreases in aggression as measured by the CBCL [88]. In a 6-week, open-label study of olanzapine in 12 children age 7-14 years with TS, aggression ratings decreased and subjects reported fewer aggressive episodes [89]. ADHD symptoms also showed significant improvements from baseline for both inattention and hyperactive/impulsivity scores [89]. Mean olanzapine dosages in these studies were 14.5 and $11.3 \mathrm{mg} /$ day, respectively, and significant weight gain occurred in both trials. A retrospective observational study of aripiprazole in 37 children and adolescents age $8-18$ years with TS demonstrated that explosive outbursts improved in $96 \%$ of subjects who completed the study on a mean daily dose of $11.69 \mathrm{mg}$. Akathisia, increased mood lability, and increased agitation were primary reasons for discontinuation. Weight gain occurred in $87 \%$ of subjects, with a mean increase of $18 \mathrm{lbs}$ over the study period [41].

\section{Other Medications}

Adjunctive anticonvulsant treatment with valproate showed efficacy in treating aggression in children with ADHD and disruptive behavior disorders who are partial responders to stimulant treatment [90]. Although treatment with valproate has demonstrated some efficacy in reducing tic symptoms, it is not recommended for routine use in children [91]. In placebocontrolled studies, lithium has demonstrated efficacy in treating conduct disorder and severe aggression [27, 92]. Augmentation of stimulants with risperidone has also demonstrated efficacy in reducing aggression, ADHD, and ODD symptoms in the recent Treatment of Severe Childhood Aggression Study [93•].

\section{Psychosocial Factors of TS and Aggression}

Environmental and psychological impacts associated with TS and tic disorders may have more adverse effects on quality of life than tics $[94,95]$. People with TS often struggle with academic, social, familial, and occupational competence [96]. Longitudinal studies show that emotional problems and problems with peer relationships tend to increase over time in children with TS [97].

Environmental factors such as harsh parenting, marital discord, and low socioeconomic status contribute to further impairment in TS $[96,98,99]$. Parents of children with TS interviewed by the 2007 National Survey of Children's Health reported greater parental aggravation [100, 101]. Parental aggravation associated with their child's comorbid psychiatric problems was also strongly linked with conduct problems 
and ASD [10, 101]. Increased parental aggravation is of clinical concern in TS, since childhood aggression has been associated with non-responsive, coercive, and unaffectionate parenting [99]. Disruption of the parentchild relationship in TS from parental aggravation and stress may possibly account for why adults with TS demonstrate higher levels of insecure attachment, anger, and hostility than healthy controls [102]. Of note, parents who reported feeling more bothered by their children exhibiting tic and rage symptoms were also more likely to punish their affected child [103]. When parents engage in conflict avoidance, failure to set limits, or harsh authoritative parenting, there may be inadvertent reinforcement of aggressive symptoms in children [104]. Family accommodation to rage attacks prompted by OCD symptoms also appears to reinforce these behaviors [105]; it is highly likely that such family accommodation to explosive outbursts in TS has a similar effect.

The negative consequences of aggressive behaviors apparently are not limited to parental involvement since children exhibiting disruptive behaviors are also commonly rejected by peers [99]. The results of such social rejection can in turn adversely influence a child's emotional regulation and social perceptions [106]. An aggression-prone child's disposition relative to their experiences with their family, peers, and community can generate maladaptive non-verbal conflict resolution, in turn, reinforcing unproductive cognitions [99]. Results from the Tourette syndrome impact survey of 211 parent-child dyads age 10-17 years show $26 \%$ reported peer victimization that was more likely in those with co-occurring psychiatric diagnosis. Victims of bullying had higher anxiety and depressive symptoms, lower quality of life, and more explosive outbursts [107].

A child's ability to accurately perceive and process external stimuli influence behavior and overall symptom severity [99]. Maladaptive cognitions or problems processing external stimuli are associated with ADHD and OCD $[72,99,108]$. Children with TS+ADHD show subtle impairments on tasks measuring emotional processing compared to healthy controls or to children with TS only, especially when processing anger items [67]. Aggressionprone children suffering from ODD or other disruptive behaviors also experience cognitive deficits that contribute to maladaptive functioning [16••, 109-111].

Co-occurring psychiatric conditions in TS contribute an even greater risk of impairment with higher levels of behavior problems and a lower quality of life [16••, 94, 112]. Children and adolescents with TS and OCD report greater psychological stress levels than healthy controls and show increased tic severity [113]. A clinical study of 60 youth aged 7-17 years with TS or CTD interviewed to assess major and minor negative and positive life events, tic severity, and comorbid disorders demonstrated a significant association between quantity of major negative life events and higher aggression scores on the Overt Aggression Scale [113]. Adult patients with TS also reported worse quality of life compared to the general population in association with $\mathrm{ADHD}, \mathrm{OCD}$, and tic severity $[16 \bullet \bullet, 69]$.

\section{Non-pharmacological Interventions}

\section{Cognitive Behavioral Therapy}

Cognitive behavioral therapy (CBT) has strong evidence supporting its application for treating TD and the cooccurring psychiatric disorder symptoms $[114,115]$. CBT functions on the premise of decreasing specific behaviors through re-education and re-learning of behavioral patterns [116]. It aims to target the tic symptoms and provide the individual with coping strategies to modify the impact of environmental factors [112]. Habit reversal therapy (HRT) is the widely accepted component CBT that integrates and implements awareness training, competing response training as well as self-monitoring, relaxation training, and contingency management as its core therapeutic principles. Awareness training teaches how to detect premonitory urges while competing response training promotes engaging in physically incompatible behaviors to prevent re-occurring tics [95, $111,112]$. Contingency management involves systematic use of reinforcements or a rewards system to promote treatment compliance and adherence [95]. CBT techniques such as external positive and negative reinforcement has been seen to impact targeted tic symptoms while providing coping and problem-solving skills beneficial in maintaining daily routines and stress reduction [112]. Individuals with ADHD and TS may not respond as well to behavioral treatment, as ADHD symptoms may impede their ability to participate in therapy [60 ${ }^{\bullet}$.

Anger control training (ACT) is a cognitive behavioral treatment that teaches children to use cognitive and behavioral coping skills to manage anger. Its efficacy for reducing disruptive behavior in children and adolescents with TS was demonstrated [55, 117]. In a study where 26 children age 11-16 years with TS and high levels of disruptive behavior were randomized to anger control training or treatment as usual (TAU), disruptive behavior rating scale score decreased by $52 \%$ in the ACT group as compared to $11 \%$ in TAU; improvements were maintained at 3-month follow-up [55]. However, while $65 \%$ of subjects in this study had explosive outbursts, only two subjects reported physical injury or destruction of property, and average scores for delinquent behavior were in the normal range [55]. The authors note that 
application of ACT may be limited by co-occurring psychiatric illness and may be less effective for more severe aggressive behaviors.

\section{Parenting Skills Training}

Parent management training is an effective behavioral treatment for children with disruptive behavior disorders, consisting of 10 sessions, which improve parental understanding of disruptive behaviors and teach strategies for improving children's compliance [95, 104, 117]. A study of 24 children with tic disorders ages 612 years with moderate disruptive behavior who were randomized to 10 sessions of structured parent management training or treatment as usual showed parent-rated disruptive behavior rating scale scores decreased by $51 \%$ in the parent management training group vs. $19 \%$ in the treatment as usual group. Parent management training demonstrated an effect size of 0.96 on disruptive behaviors, which approximates the effect size demonstrated in children with ODD [104]. Behavioral reinforcement at home is essential to treatment compliance. This requires active parental involvement in treatment parameters and increasing parental awareness through psychoeducation [100, 104, 112, 118]. Parents need to be encouraged to hold realistic goals and appropriate expectations for their child with TS [99]. It has been shown that providing parents and other primary caregivers with support and resources reduces parental anxiety related to their child's tic and associated psychiatric symptoms [16••, 112].

\section{Conclusion}

Aggression symptoms are very common in TS that is complicated by psychiatric comorbidities. When present, these symptoms cause considerable morbidity with significant negative consequences for both individual and family. Current evidence suggests that tic severity and psychiatric comorbidity are associated with aggressive symptoms in TS. However, it is likely that aggressive symptoms result from a complex interplay of environmental, genetic, neurocognitive, and neuropsychiatric factors. Larger, more rigorously designed clinical studies are necessary to identify specific risk factors and to help untangle overlapping symptoms of psychiatric comorbidity and potential short and longer-term medication side effect with behavioral toxicity. There is good evidence for efficacy of some non-pharmacological treatments but evidence for current psychotropic medication interventions remains limited and requires further investigation.

\section{Compliance with Ethics Guidelines}

Conflict of Interest Cathy L. Budman is a speaker for the National Tourette Syndrome Association-Center for Disease Control (CDC) Partnership and receives funding to conduct clinical trials from the following pharmaceutical companies: Psyadon, Otsuka Pharmaceutical, AstraZeneca, Auspex, and Synchroneuron Inc.

Miri Rosen and Sana Shad declare that they have no conflict of interest.

Human and Animal Rights and Informed Consent This article does not contain any studies with human or animal subjects performed by any of the authors.

\section{References}

Papers of particular interest, published recently, have been highlighted as:

- Of importance

•- Of major importance

1. American Psychiatric Association. Diagnostic and statistical manual of mental disorders. 5th ed. Arlington: American Psychiatric Publishing; 2013.

2. Roberton MM, Eapen V, Cavanna AE. The international prevalence, epidemiology, and clinical phenomenology of Tourette syndrome: a cross-cultural perspective. J Psychosom Res. 2009;67: 475-83. doi:10.1016/j.jpsychores.2009.07.010.

3. Knight T, Steeves T, Day L, et al. Prevalence of tic disorders: a systematic review and meta-analysis. Pediatr Neurol. 2012;47:7790. doi:10.1016/j.pediatrneurol.2012.05.002.

4. Scharf JM, Miller LL, Gauvin CA, Alabiso J, et al. Population prevalence of Tourette syndrome: a systematic review and metaanalysis. Mov Disord. 2015;30:221-8. doi:10.1002/mds.26089.

5. Kraft JT, Dalsgaard S, Obel C, et al. Prevalence and clinical correlates of tic disorders in a community sample of school-age children. Eur Child Adolesc Psychiatry. 2012;21:5-13. doi:10.1007/ s00787-011-0223-z.

6. Rizzo R, Gulisano M, Calì PV, et al. Long term clinical course of Tourette syndrome. Brain Dev. 2012;34:667-73. doi:10.1016/j. braindev.2011.11.006.

7. Leckman J, King R, Bloch M. Clinical features of Tourette syndrome and tic disorders. J Obsessive Compuls Relat Disord. 2014;3:372-9. doi:10.1016/j.jocrd.2014.03.004. This is an excellent review of TS and related disorders.

8. Robertson MM. The Gilles de la Tourette syndrome: the current status. Arch Dis Child Educ Pract Ed. 2012;97:166-75. doi:10. 1136/archdischild-2011-300585.

9. Robertson MM, Eapen V. Wither the relationship between etiology and phenotype in Tourette syndrome? In: Leckman JF, Martino D, editors. Tourette syndrome. New York: Oxford University Press; 2013. p. 361-94. doi:10.1093/med/9780199796267.003. 0016.

10. Bitsko RH, Holbrook JR, Visser SN, et al. A national profile of Tourette syndrome, 2011-2012. J Dev Behav Pediatr. 2014;35: 317-22. doi:10.1097/DBP.0000000000000065.

11.• Hirschtritt ME, Lee PC, Pauls DL, et al. Lifetime prevalence, age of risk, and genetic relationships of comorbid psychiatric disorders in Tourette syndrome. JAMA Psychiatry. 2015;72:325-33. doi:10. 1001/jamapsychiatry.2014.2650. This is a landmark study of psychiatric comorbidity in TS, the largest to date with rigorous methodology. 
12. Dooley JM, Brna PM, Gordon KE. Parent perceptions of symptom severity in Tourette's syndrome. Arch Dis Child. 1999;81: 440-1. doi:10.1136/adc.81.5.440.

13. Budman CL, Rockmore L, Stokes J, et al. Clinical phenomenology of episodic rage in children with Tourette syndrome. J Psychosom Res. 2003;55:59-65. doi:10.1016/S0022-3999(02) 00584-6.

14. Kadesjö B, Gillberg C. Tourette's disorder: epidemiology and comorbidity in primary school children. J Am Acad Child Adolesc Psychiatry. 2000;39:548-55.

15. Ghanizadeh A, Mosallaei S. Psychiatric disorders and behavioral problems in children and adolescents with Tourette syndrome. Brain Dev. 2009;31:15-9. doi:10.1016/j.braindev.2008.03.010.

16.• Robertson MM, Cavanna AE, Eapen V. Gilles de la Tourette syndrome and disruptive behavior disorders: prevalence, associations, and explanation of the relationships. J Neuropsychiatry Clin Neurosci. 2015;27:33-41. doi:10.1176/appi.neuropsych. 13050112. This is an excellent overview including original data on TS and disruptive behaviors including impulsive aggression.

17. Freeman RD, Fast $\mathrm{D}, \mathrm{Burd} \mathrm{L}$, et al. An international perspective on Tourette syndrome: selected findings from 3,500 individuals in 22 countries. Dev Med Child Neurol. 2000;42:436-47. doi:10. 1017/S0012162200000839.

18. Cavanna AE, Selvini C, Luoni C, et al. Measuring anger expression in young patients with Tourette syndrome. Child Health Care. 2014. doi:10.1080/02739615.2014.896216. This is an important clinical study of aggressive symptoms and explosive outbursts in TS.

19. Wright A, Rickards H, Cavanna AE. Impulse-control disorders in Gilles de la Tourette syndrome. J Neuropsychiatry Clin Neurosci. 2012;24:16-27. doi:10.1176/appi.neuropsych.10010013.

20. Daniels E, Mandleco B, Luthy KE. Assessment, management, and prevention of childhood temper tantrums. J Am Acad Nurse Pract. 2012;24:569-73. doi:10.1111/j.1745-7599.2012.00755.x.

21. Wakschlag LS, Choi SW, Carter AS, et al. Defining the developmental parameters of temper loss in early childhood: implications for developmental psychopathology. J Child Psychol Psychiatry. 2012;53:1099-108. doi:10.1111/j.1469-7610.2012.02595.x.

22. Hong JS, Tillman R, Luby JL. Disruptive behavior in preschool children: distinguishing normal misbehavior from markers of current and later childhood conduct disorder. J Pediatr. 2015;166: 723-30. doi:10.1016/j.jpeds.2014.11.041.

23. Victoroff J. Human aggression. In: Kaplan HI, Sadock BJ, editors. Comprehensive textbook of psychiatry. 9th ed. Baltimore: Lipincott, Williams \& Wilkins; 2009. p. 2671-703.

24. White BA, Jarrett MA, Ollendick TH. Self-regulation deficits explain the link between reactive aggression and internalizing and externalizing behavior problems in children. J Psychopathol Behav Assess. 2013;35:1-9. doi:10.1007/s10862-012-9310-9.

25. Rosell DR, Siever LJ. The neurobiology of aggression and violence. CNS Spectr. 2015;20:254-79. doi:10.1017/ S109285291500019X.

26. Liu J. Early health risk factors for violence: conceptualization, evidence, and implications. Aggress Violent Behav. 2011;16:63-73.

27. Weisbrot DM, Ettinger AB. Aggression and violence in mood disorders. Child Adolesc Psychiatr Clin N Am. 2002;11:649-71.

28. Roy AK, Lopes V, Klein RG. Disruptive mood dysregulation disorder: a new diagnostic approach to chronic irritability in youth. Am J Psychiatry. 2014;171:918-24. doi:10.1176/appi.ajp. 2014.13101301.

29. Lennox BR, Lennox GG. Mind and movement: the neuropsychiatry of movement disorders. J Neurol Neurosurg Psychiatry. 2002;72:28-31.
30. Roffman J, Eisenberg T, Stern T. Neuropsychiatric phenomena associated with movement disorders. Prim Care Companion J Clin Psychiatry. 2006;8:39-42.

31. Murphy TK, Gerardi DM, Leckman JF. Pediatric acute-onset neuropsychiatric syndrome. Psychiatr Clin North Am. 2014;37:35374. doi:10.1016/j.psc.2014.06.001.

32. Liu J, Lewis G, Evans L. Understanding aggressive behaviour across the lifespan. J Psychiatr Ment Health Nurs. 2013;20:15668. doi:10.1111/j.1365-2850.2012.01902.x.

33. Shiina A. Neurobiological basis of reactive aggression: a review. Int J Forensic Sci Pathol. 2015;3:94-8.

34. Mol Debes NM, Hjalgrim H, Skov L. Validation of the presence of comorbidities in a Danish clinical cohort of children with Tourette syndrome. J Child Neurol. 2008;23:1017-27. doi:10.1177/ 0883073808316370

35. Chen K, Budman CL, Herrera LD, et al. Prevalence and clinical correlates of explosive outbursts in Tourette syndrome. Psychiatry Res. 2013;205:269-75. doi:10.1016/j.psychres.2012.09.029. This is an important study examining prevalence and clinical features of explosive outbursts in two different populations.

36. Kurlan R, Daragjati C, Como PG, et al. Non-obscene complex socially inappropriate behavior in Tourette's syndrome. J Neuropsychiatry Clin Neurosci. 1996;8:311-7.

37. Eddy CM, Cavanna AE. On being your own worst enemy: an investigation of socially inappropriate symptoms in Tourette syndrome. J Psychiatr Res. 2013;47:1259-63. doi:10.1016/j. jpsychires.2013.05.019. This study highlights clinical prevalence of an often overlooked and clinically relevant clinical symptom that may contribute to aggressive behaviors in TS.

38. Kano Y, Ohta M, Nagai Y, et al. Rage attacks and aggressive symptoms in Japanese adolescents with Tourette syndrome. CNS Spectr. 2008;13:325-32.

39. Budman CL, Bruun RD, Park KS, et al. Rage attacks in children and adolescents with Tourette's disorder: a pilot study. J Clin Psychiatry. 1998;59:576-80.

40. Budman CL, Bruun RD, Park KS, et al. Explosive outbursts in children with Tourette's disorder. J Am Acad Child Adolesc Psychiatry. 2000;39(10):1270-6.

41. Budman CL, Coffey BJ, Shechter R, et al. Aripiprazole in children and adolescents with Tourette disorder with and without explosive outbursts. J Child Adolesc Psychopharmacol. 2008;18:509-15. doi:10.1089/cap.2007.061.

42. Stephens RJ, Sandor P. Aggressive behaviour in children with Tourette syndrome and comorbid attention-deficit hyperactivity disorder and obsessive-compulsive disorder. Can J Psychiatry. 1999;44:1036-42.

43. Zhu Y, Leung KM, Liu PZ, et al. Comorbid behavioural problems in Tourette's syndrome are positively correlated with the severity of tic symptoms. Aust N Z J Psychiatry. 2006;40:67-73.

44. Cavanna AE, Critchley HD, Orth M, et al. Dissecting the Gilles de la Tourette spectrum: a factor analytic study on 639 patients. J Neurol Neurosurg Psychiatry. 2011;82:1320-3. doi:10.1136/ jnnp.2010.225029.

45.• Frank MC, Piedad J, Rickards H, et al. The role of impulse control disorders in Tourette syndrome: an exploratory study. J Neurol Sci. 2011;310:276-8. doi:10.1016/j.jns.2011.06.032. This is an important study exploring different types of impulse control disorders in TS and their clinical correlates including intermittent explosive disorder and impulsive aggression.

46. Alsobrook JP, Pauls DL. A factor analysis of tic symptoms in Gilles de la Tourette's syndrome. Am J Psychiatry. 2002;159: 291-6.

47. Robertson MM, Trimble MR, Lees AJ. Self-injurious behaviour and the Gilles de la Tourette syndrome: a clinical study and review of the literature. Psychol Med. 1989;19:611-25. 
48. Mathews CA, Waller J, Glidden D, et al. Self injurious behaviour in Tourette syndrome: correlates with impulsivity and impulse control. J Neurol Neurosurg Psychiatry. 2004;75:1149-55. doi: 10.1136/jnnp.2003.020693.

49. Bhidayasiri R, Tarsy D. Tourette's syndrome: self-injurious behavior. In: Movement disorders: a video atlas. Humana Press: New York, 2012. p. 242-243. doi:10.1007/978-1-60327-426-5 111.

50. Robertson MM, Stern JS. Gilles de la Tourette syndrome: symptomatic treatment based on evidence. Eur Child Adolesc Psychiatry. 2000;9:160-75.

51. Freeman RD. Tourette Syndrome International Database Consortium. Tic disorders and ADHD: answers from a worldwide clinical data-set on Tourette syndrome. Eur Child Adolesc Psychiatry. 2007;16:15-23. doi:10.1007/s00787-007-1003-7.

$52 . \bullet$ Cox JH, Cavanna AE. Irritability symptoms in Gilles de la Tourette syndrome. J Neuropsychiatry Clin Neurosci. 2015;27: 42-7. doi:10.1176/appi.neuropsych.13060143. This is an excellent study of a clinically important symptoms relevant to explosive outbursts and aggressive symptoms in TS.

53.• Rizzo R, Gulisano M, Pellico A, et al. Tourette syndrome and comorbid conditions a spectrum of different severities and complexities. J Child Neurol. 2014;29:1383-9. doi:10.1177/ 0883073814534317 . This review covers many important aspects of psychiatric comorbidities relevant to understanding aggressive symptoms in TS.

54. Eddy CM, Rickards HE, Cavanna AE. Treatment strategies for tics in Tourette syndrome. Ther Adv Neurol Disord. 2011;4:2545. doi:10.1177/1756285610390261.

55. Sukhodolsky DG, Vitulano LA, Carroll DH, et al. Randomized trial of anger control training for adolescents with Tourette's syndrome and disruptive behavior. J Am Acad Child Adolesc Psychiatry. 2009;48:413-21. doi:10.1097/CHI. 0b013e3181985050.

56.• Sukhodolsky DG, Scahill L, Zhang H, et al. Disruptive behavior in children with Tourette's syndrome: association with ADHD comorbidity, tic severity, and functional impairment. J Am Acad Child Adolesc Psychiatry. 2003;42:98-105. This study provides evidence for a valuable non-pharmacological intervention for treatment of explosive anger in TS.

57. Robertson MM. Mood disorders and Gilles de la Tourette's syndrome: an update on prevalence, etiology, comorbidity, clinical associations, and implications. J Psychosom Res. 2006;61: 349-58.

58. Scharf JM, Miller LL, Mathews CA, et al. Prevalence of Tourette syndrome and chronic tics in the population-based Avon longitudinal study of parents and children cohort. J Am Acad Child Adolesc Psychiatry. 2012;51:192-201. doi:10.1016/j.jaac.2011. 11.004.

59. Cavanna AE, Eddy C, Rickards HE. Cognitive functioning in Tourette syndrome. Discov Med. 2009;8:191-5.

60. El Malhany N, Gulisano M, Rizzo R, et al. Tourette syndrome and comorbid ADHD: causes and consequences. Eur J Pediatr. 2015;174:279-88. doi:10.1007/s00431-014-2417-0. This is an excellent update on the relationship between TS and ADHD including role of ADHD in disruptive behaviors.

61. Stephens RJ, Chung SA, Jovanovic D, et al. Relationship between polysomnographic sleep architecture and behavior in medicationfree children with TS, ADHD, TS and ADHD, and controls. J Dev Behav Pediatr. 2013;34:688-96. doi:10.1097/DBP. 0000000000000012.

62. Spencer T, Biederman J, Harding M, et al. Disentangling the overlap between Tourette's disorder and ADHD. J Child Psychol Psychiatry. 1998;39:1037-44.

63. Cardona F, Romano A, Bollea L, et al. Psychopathological problems in children affected by tic disorders. Eur Child Adolesc Psychiatry. 2004;13:166-71.
64. Scahill L, Williams S, Schwab-Stone M, et al. Disruptive behavior problems in a community sample of children with tic disorders. Adv Neurol. 2006;99:184-90.

65. Rizzo R, Curatolo P, Gulisano M, et al. Disentangling the effects of Tourette syndrome and attention deficit hyperactivity disorder on cognitive and behavioral phenotypes. Brain Dev. 2007;29: 413-20.

66. Pollak Y, Benarroch F, Kanengisser L, et al. Tourette syndromeassociated psychopathology: roles of comorbid attention-deficit hyperactivity disorder and obsessive-compulsive disorder. J Dev Behav Pediatr. 2009;30:413-9.

67. Drury H, Channon S, Barrett R, et al. Emotional processing and executive functioning in children and adults with Tourette's syndrome. Child Neuropsychol. 2012;18:281-98. doi:10.1080/ 09297049.2011.613811

68. Hovik KT, Egeland J, Isquith PK, et al. Distinct patterns of everyday executive function problems distinguish children with Tourette syndrome from children with ADHD or autism spectrum disorders. J Atten Disord. 2014;1-13 doi:10.1177/ 1087054714550336. This is an important update on neuropsychological findings associated with TS and ADHD that are relevant to impulsive aggression.

69. Haddad AD, Umoh G, Bhatia V, et al. Adults with Tourette's syndrome with and without attention deficit hyperactivity disorder. Acta Psyciatr Scand. 2009;120:299-307. doi:10.1111/j.16000447.2009.01398.x.

70. Thériault MCG, Lespérance P, Achim A, et al. ODD irritability is associated with obsessive-compulsive behavior and not ADHD in chronic tic disorders. Psychiatry Res. 2014;220:447-52. doi:10. 1016/j.psychres.2014.07.039.

71. Coffey BJ, Biederman J, Geller DA, et al. Distinguishing illness severity from tic severity in children and adolescents with Tourette's disorder. J Am Acad Child Adolesc Psychiatry. 2000;39:556-61.

72. Eddy CM, Cavanna AE. Tourette syndrome and obsessive compulsive disorder: compulsivity along the continuum. J Obsessive Compuls Relat Disord. 2014;3:363-71.

73. Kano Y, Kono T, Matsuda N, et al. The impact of tics, obsessivecompulsive symptoms, and impulsivity on global functioning in Tourette syndrome. Psychiatry Res. 2015;226:156-61. doi:10. 1016/j.psychres.2014.12.041. This study shows the relationship of decreased global functioning with aggressive symptoms in a Japanese cohort with TS.

74. • Storch EA, Jones AM, Lack CW, et al. Rage attacks in pediatric obsessive-compulsive disorder: phenomenology and clinical correlates. J Am Acad Child Adolesc Psychiatry. 2012;51:582-92. doi:10.1016/j.jaac.2012.02.016. This important study emphasizes the likely role of family accommodation in sustaining aggressive behaviors in the home and also examines phenomenology of rage in $\mathrm{OCD}$.

75. Brem S, Grünblatt E, Drechsler R, et al. The neurobiological link between OCD and ADHD. Atten Defic Hyperact Disord. 2014;6: 175-202. doi:10.1007/s12402-014-0146-x.

76. Cavanna AE, Rickards $\mathrm{H}$. The psychopathological spectrum of Gilles de la Tourette syndrome. Neurosci Biobehav Rev. 2013;37:1008-15. doi:10.1016/j.neubiorev.2012.10.011.

77. Eapen V, Fox-Hiley P, Banerjee S, et al. Clinical features and associated psychopathology in a Tourette syndrome cohort. Acta Neurol Scand. 2004;109:255-60.

78. Storch EA, Hanks CE, Mink JW, et al. Suicidal thoughts and behaviors in children and adolescents with chronic tic disorders. Depress Anxiety. 2015. doi:10.1002/da.22357.

79. Serajee FJ, Huq AM. Advances in Tourette syndrome: diagnoses and treatment. Pediatr Clin North Am. 2015;62:687-701. doi:10. 1016/j.pcl.2015.03.007. 
80. Kurlan RM. Treatment of Tourette syndrome. Neurotherapeutics. 2014;11:161-5. doi:10.1007/s13311-013-0215-4. This is a nice update on treatment of TS

81. Riddle MA, King RA, Hardin MT, et al. Behavioral side effects of fluoxetine in children and adolescents. J Child Adolesc Psychopharmacol. 1991;1:193-8.

82. Gadow KD, Nolan E, Sprafkin J, et al. School observations of children with attention-deficit hyperactivity disorder and comorbid tic disorder: effects of methylphenidate treatment. J Dev Behav Pediatr. 1995;16:167-76.

83. Connor DF, Glatt SJ, Lopez ID, et al. Psychopharmacology and aggression. I: a meta-analysis of stimulant effects on overt/covert aggression-related behaviors in ADHD. J Am Acad Child Adolese Psychiatry. 2002;41:253-61. doi:10.1097/00004583-20020300000004.

84. Gadow KD, Sverd J, Nolan EE, et al. Immediate-release methylphenidate for ADHD in children with comorbid chronic multiple tic disorder. J Am Acad Child Adolesc Psychiatry. 2007;46:840-8.

85. Gadow KD, Nolan EE, Sverd J, et al. Methylphenidate in children with oppositional defiant disorder and both comorbid chronic multiple tic disorder and ADHD. J Child Neurol. 2008;23:981-90. doi: $10.1177 / 0883073808315412$.

86.• Murphy TK, Lewin AB, Storch EA, et al. Practice parameter for the assessment and treatment of children and adolescents with tic disorders. J Am Acad Child Adolesc Psychiatry. 2013;52:134159. doi:10.1016/j.jaac.2013.09.015. This is the most up-to-date treatment parameters for TS in the United States and includes discussion of combined therapies for psychiatric comorbidities.

87. Sandor P, Stephens RJ. Risperidone treatment of aggressive behavior in children with Tourette syndrome. J Clin Psychopharmacol. 2000;20:710-2.

88. Stephens RJ, Bassel C, Sandor P. Olanzapine in the treatment of aggression and tics in children with Tourette's syndrome - a pilot study. J Child Adolesc Psychopharmacol. 2004;14:255-66.

89. McCracken JT, Suddath R, Chang S, et al. Effectiveness and tolerability of open label olanzapine in children and adolescents with Tourette syndrome. J Child Adolesc Psychopharmacol. 2008;18: 501-8. doi:10.1089/cap.2007.135.

90. Blader JC, Schooler NR, Jensen PS, et al. Adjunctive divalproex versus placebo for children with ADHD and aggression refractory to stimulant monotherapy. Am J Psychiatry. 2009;166:1392-401. doi:10.1176/appi.ajp.2009.09020233.

91. Yang CS, Zhang LL, Lin YZ, et al. Sodium valproate for the treatment of Tourette's syndrome in children: a systematic review and meta-analysis. Psychiatry Res. 2015;226:411-7. doi:10.1016/ j.psychres.2014.08.058.

92. Pine DS, Cohen E. Therapeutics of aggression in children. Paediatr Drugs. 1999;1:183-96.

93. Gadow KD, Arnold LE, Molina BS, et al. Risperidone added to parent training and stimulant medication: effects on attention-deficit/hyperactivity disorder, oppositional defiant disorder, conduct disorder, and peer aggression. J Am Acad Child Adolesc Psychiatry. 2014;53:948-59. doi:10.1016/j.jaac.2014.05.008. This is an important combined clinical treatment trial for TS with comorbid ADHD, ODD, CD and aggressive symptoms and merits further follow-up in a larger sample.

94. Espil FM, Capriotti MR, Conelea CA, et al. The role of parental perceptions of tic frequency and intensity in predicting tic-related functional impairment in youth with chronic tic disorders. Child Psychiatry Hum Dev. 2014;45:657-65. doi:10.1007/s10578-0130434-2.

95. McGuire JF, Arnold E, Park JM, et al. Living with tics: reduced impairment and improved quality of life for youth with chronic tic disorders. Psychiatry Res. 2015;225:571-9. doi:10.1016/j. psychres.2014.11.045.
96. Liu S, Zheng L, Zheng X, et al. The subjective quality of life in young people with Tourette syndrome in China. J Atten Disord. 2014;1-7. doi:10.1177/1087054713518822.

97. Hoekstra PJ, Lundervold AJ, Lie SA, et al. Emotional development in children with tics: a longitudinal population-based study. Eur Child Adolesc Psychiatry. 2013;22:185-92. doi:10.1007/ s00787-012-0337-y.

98. Walkup JT. Epigenetic and environmental risk factors in Tourette syndrome. Adv Neurol. 2001;85:273-9.

99. Lochman JE, Powell NP, Boxmeyer CL, et al. Cognitivebehavioral therapy for externalizing disorders in children and adolescents. Child Adolesc Psychiatr Clin N Am. 2011;20:305-18. doi:10.1016/j.chc.2011.01.005.

100. Schieve LA, Boulet SL, Kogan MD, et al. Parenting aggravation and autism spectrum disorders: 2007 National Survey of Children's Health. Disabil Health J. 2011;4:143-52. doi:10. 1016/j.dhjo.2010.09.002.

101. Robinson LR, Bitsko RH, Schieve LA, et al. Tourette syndrome, parenting aggravation, and the contribution of co-occurring conditions among a nationally representative sample. Disabil Health J. 2013;6:26-35. doi:10.1016/j.dhjo.2012.10.002.

102. Ghanizadeh A, Mohammadi MR, Dehbozorgi GR. Children and their parent's perceptions of symptom severity and treatment preference for Tourette syndrome. Iran J Psychiatry. 2010;5:93-6.

103. Scahill L, Sukhodolsky DG, Bearss K, et al. Randomized trial of parent management training in children with tic disorders and disruptive behavior. J Child Neurol. 2006;21:650-6.

104. Stewart SE. Rage takes center stage: focus on an underappreciated aspect of pediatric obsessive-compulsive disorder. J Am Acad Child Adolesc Psychiatry. 2012;51:569-71. doi:10.1016/j.jaac. 2012.04.002.

105. Dehning S, Burger MB, Krause D, et al. Tourette syndrome is associated with insecure attachment and higher aggression. Int J Neurosci. 2014. doi:10.3109/00207454.2014.951040.

106. Pardini DA, Barry TD, Barth JM, et al. Self-perceived social acceptance and peer social standing in children with aggressivedisruptive behaviors. Soc Dev. 2006;15:46-64.

107. Zinner SH, Conelea CA, Glew GM, et al. Peer victimization in youth with Tourette syndrome and other chronic tic disorders. Child Psychiatry Hum Dev. 2012;43:124-36. doi:10.1007/ s10578-011-0249-y.

108. Lavoie ME, O'Connor KP. Neurocognitive aspects of Tourette syndrome and related disorders, when things go wrong - diseases and disorders of the human brain. In: T Mantamadiotis, editor. InTech; 2012. doi:10.5772/36744.

109. Conelea CA, Woods DW, Zinner SH, et al. Exploring the impact of chronic tic disorders on youth: results from the Tourette Syndrome Impact Survey. Child Psychiatry Hum Dev. 2011;42: 219-42.

110. Conelea CA, Woods DW, Zinner SH, et al. The impact of Tourette syndrome in adults: results from the Tourette Syndrome Impact Survey. Community Ment Health J. 2013;49:110-20.

111. McGuire JF, Piacentini J, Brennan EA, et al. A meta-analysis of behavior therapy for Tourette syndrome. J Psychiatr Res. 2014;50: 106-12. doi:10.1016/j.jpsychires.2013.12.009.

112. Bennett S, Keller AE, Walkup JT. The future of tic disorder treatment. Ann N Y Acad Sci. 2013;1304:32-9. doi:10.1111/nyas. 12296.

113. Steinberg T, Shmuel-Baruch S, Horesh N, et al. Life events and Tourette syndrome. Compr Psychiatry. 2013;54:467-73. doi:10. 1016/j.comppsych.2012.10.015.

114. Verdellen C, van de Griendt J, Hartmann A, et al. European clinical guidelines for Tourette syndrome and other tic disorders. Part III: Behavioural and psychosocial interventions. Eur Child Adolesc Psychiatry. 2011;20:197-207. doi:10.1007/s00787-0110167-3. 
115. Wilhelm S, Peterson AL, Piacentini J, et al. Randomized trial of behavior therapy for adults with Tourette syndrome. Arch Gen Psychiatry. 2012;69:795-803. doi:10.1001/archgenpsychiatry. 2011.1528

116. Leclerc J, O'Connor KP, Forget J, et al. Behavioral program for managing explosive outbursts in children with Tourette syndrome. J Dev Phys Disabil. 2011;23:33-47. doi:10.1007/s10882-0109213-1.
117. Sukhodolsky DG, Scahill L. Cognitive-behavioral therapy for anger and aggression in children and adolescents. New York: Guilford Press; 2012.

118. Vural P, Akkaya C, Kucukparlak I, et al. Psychodramatic group psychotherapy as a parental intervention in attention deficit hyperactivity disorder: a preliminary study. Arts Psychotherapy. 2014;41:233-9. doi:10.1016/j.aip.2014.02.004. 